\title{
Comparison of Left Ventricular Systolic Function by 2D Echocardiography between Diabetic and Non-Diabetic Patients undergoing Percutaneous Coronary Intervention in Non-ST elevated Myocardial Infarction
}

\author{
MSI TIPU CHOWDHURY, HARISUL HOQUE, MANZOOR MAHMOOD, KHURSHED AHMED, \\ MD. MUKHLESUR RAHMAN, FAKHRUL ISLAM KHALED, KHALED MD. IQBAL, \\ PRITAM KUMAR GACHCHHADER, NILUFAR FATEMA, SM MUSTAFA ZAMAN \\ Department of Cardiology,Bangabandhu Sheikh Mujib Medical University, Dhaka.
}

Address of Correspondance: Dr. MSI Tipu Chowdhury, Resident, Department of Cardiology, Bangabandhu Sheikh Mujib Medical University (BSMMU), Dhaka, Bangladesh. Email: dr.tipuchowdhury@gmail.com

\begin{abstract}
:
Background: Long term mortality is higher in patients with Non-ST-segment elevated myocardial infarction (NSTEMI) than those with STEMI. In diabetic patients with NSTEMI are at high risk for subsequent cardiovascular events. But, the widespread use of drug eluting stents(DES) will further improve outcomes in patients with diabetes undergoing early percutaneous coronary intervention(PCI). Objective: The aim of the study was to determine the changes in left ventricular (LV) systolic function after successful PCI in NSTEMI diabetic patients compared with non-diabetic patients. Methods: From April 2017 to March 2018, this comparative clinical study was carried out in the Department of Cardiology, University Cardiac Center, Bangabandhu Sheikh Mujib Medical University (BSMMU), Dhaka, Bangladesh. 30 diabetic and 34 nondiabetic patients with NSTEMI undergoing Percutaneous coronary intervention were included in the study. Successful PCI with drug eluting stent was performed for all patients. 2-Dimensional echocardiography was done at baseline, at discharge following PCI and 3 months thereafter to measure the LV systolic functions and compare them between diabetics and non-diabetics at all levels of evaluation to assess the outcome of intervention. Results: At baseline LVEF was somewhat lower in diabetic group than that in non-diabetic group. Number of segments with abnormal wall motion (WMA) was higher in the diabetics compared to the non-diabetics. While the LVEDV, LVIDd and LVIDs were significantly greater in the former group than those in the latter group, the LVESV was no different between the groups. At discharge, no significant improvement was observed in either group following PCI in terms of LVEF, number of segments with WMA, LVIDd and LVIDs. However, both LVEDV and LVESV reduced effectively in both groups with decrease of LVESV being more marked in the nondiabetics compared to that in diabetics $(p=0.018)$. However, 3 months after PCI, LVEF improved $8.4 \pm 1.2 \%$ in diabetics and $7.9 \pm 1.2 \%$ in non diabetics but the difference of this improvement between two groups was not statistically significant $(p=0.631)$. Similarly baseline to 3 months after PCI LVIDs decreases in diabetics $5.7 \pm 1.9 \%$ and in non diabetics $4.8 \pm 1.1 \%$ but the difference between these two groups was not significant $(p=$ $0.201)$. Diabetic patients more often required 2 stents $(p=0.30)$, although the diameter and length of the stents did not differ between the study groups. Conclusion: Our study demonstrated that improvement of the parameters of left ventricular systolic function after using of drug eluting stent in diabetic patients with NSTEMI was not inferior to the non diabetic group under same condition. So, indications of PCI with drug eluting stent may be extended in diabetic patient with NSTEMI.
\end{abstract}

Key words: Non-ST-segment elevated myocardial infarction (NSTEMI), Diabetes Mellitus (DM), Percutaneous Coronary Intervention (PCI), Left Ventricular ejection fraction (LVEF).

University Heart Journal 2018; 14(2): 47-52

Introduction:

Worldwide, more than 4 million people each year are estimated to have a non-ST-segment elevated myocardial infarction (NSTEMI) and long term mortality is higher in patients with NSTEMI than those with STEMI. ${ }^{1,2}$ In total, $15-20 \%$ of patients who undergo coronary revascularization are diabetic ${ }^{3}$.The long-term results of percutaneous coronary intervention (PCI) and coronary artery bypass graft (CABG) are less favorable in diabetic patients. ${ }^{4}$ This outcome is most likely due to a faster 
progression of atherosclerosis and a higher rate of restenosis ${ }^{5}$. While the use of stents has improved the short and long term outcomes of PCI in diabetic patients. ${ }^{6}$

Diabetic patients with non-ST-segment elevation acute coronary syndromes are at high risk for subsequent cardiovascular events. ${ }^{7}$ At the same time, however, they derive greater benefit than non-diabetic counter parts from an aggressive acute phase management based on platelet glycoprotein IIb/IIIa receptor antagonists and an early invasive strategy. ${ }^{8}$ Despite the benefit, these treatment modalities remain underutilized among patients with diabetes mellitus. The widespread use of drug eluting stents will further improve outcomes in patients with diabetes undergoing early percutaneous revascularization. ${ }^{7,8}$ As the improvement in LV systolic function is associated with better outcome and functional capacity, the existence of DM did not have negative effect in left ventricular ejection fraction (LVEF) improvement after angioplasty. ${ }^{9}$

There are many studies showed, long term clinical outcome \& survival benefit of drug eluting stent in patients with diabetes compared to non diabetics. Few studies have compared the echocardiographic systolic parameters between diabetics and non-diabetic patients with STEMI which showed that PCI on left coronary artery (LAD) or left circumflex artery (LCx) in STEMI patients induced further improvement in LVEF. But, there is lack of study to compare left ventricular systolic function between diabetic and non diabetic patients with NSTEMI undergoing PCI. Therefore, this study was designed to determine the changes in left ventricular systolic function after successful PCI with drug-eluting stenting in LAD or LCx or right coronary artery(RCA) after NSTEMI in diabetic patients compared with non-diabetic patients.

\section{Methods:}

\section{Patients}

From April 2017 to March 2018, this study was carried out in the Department of Cardiology, University Cardiac Center, Bangabandhu Sheikh Mujib Medical University (BSMMU), Shahbag, Dhaka, Bangladesh. 30 diabetic and 34 non-diabetic patients with non-STEMI undergoing percutaneous coronary intervention were included in the study. All patients were assessed by 2D echocardiography Then, alterations in the echocardiographic variables after the procedure were compared between the two groups.

The study was performed according to the guidelines of the Helsinki Declaration and was approved by the
University ethical committee. Written informed consent was obtained from all of the patients. Patients who were diagnosed as NSTEMI included in this study. However, patients with chronic stable angina, unstable angina, ST elevated myocardial infarction, congenital heart disease, significant valvular heart disease (equal or more than moderate severity), cardiomyopathy and atrial fibrillation, systemic diseases, such as cancer, collagen vascular diseases or amyloidosis, renal impairment were excluded from the study.

\section{Echocardiography}

Transthoracic 2D echocardiography was performed at baseline within $24 \mathrm{~h}$ before PCI, at discharge and was repeated 3 months after PCI for all patients by a Vivid E9 version: 113 (GE Healthcare, Norway), 1.5-4.6MHz transducer. All of the measurements represent the average of three consecutive beats between normal heart rate ranges, 60-100 beat per minute. The images were stored on a hard disk for better offline measurements, and the results were confirmed by an echocardiographer who was blind to the patient's information. Patients with a poor echo window were excluded from the study.

Estimation of the LV systolic dimensions were derived from the LV minor-axis dimensions with the transducer in the parasternal position. So that the cursor was perpendicular to the interventricular septum and posterior wall at the mid-papillary muscle level. The EF and wall motion abnormalities (WMA) were determined. The EF was defined as the end diastolic volume minus the end systolic volume divided by the end diastolic volume frombiplane apical two and four chamber views using a modified Simpson's technique.

Blood samples were obtained during fasting, and the levels of plasma glucose, total cholesterol (T-chol), high density lipoprotein (HDL)-chol, low density lipoprotein (LDL)-chol, and triglycerides (TG) were measured. The systolic and diastolic blood pressures were measured after $5 \mathrm{~min}$ of rest. The height and weight were measured, and the body mass index (BMI) was calculated as the body weight divided by the height squared. Hypertension was defined as a systolic blood pressure $\geq 140 \mathrm{mmHg}$, a diastolic blood pressure $\geq 90 \mathrm{mmHg}^{10}$ or the requirement for antihypertensive medication. The diabetes mellitus (DM) was defined according to the criteria of the American Diabetes Association ${ }^{11}$ or the requirement for insulin or oral hypoglycemic drugs. A family history of coronary artery disease (CAD) was defined as having a first-degree relative (a male $<55$ years or female $<65$ years) with a history of myocardial infarction, coronary 
revascularization, or sudden death. ${ }^{12}$ The history of smoking was determined by a faceto-face questionnaire.

Coronary angiography was performed for all of the patients using a cardiac angiography system (Siemens AG, Medical Solutions, Erlangen, Germany), and they all underwent PCI. PCI was performed by standard techniques, and newer generation of drug eluting stents were used.

Procedural success was defined as the successful deployment of the stent and residual stenosis of less than $10 \% .{ }^{13}$ Procedural anticoagulation was achieved with unfractionated heparin; glycoprotein IIb/IIIa inhibitors were used whenever needed. Patients received $180 \mathrm{mg}$ of Ticagrelor before the intervention. Thereafter, $75 \mathrm{mg}$ of aspirin daily and $90 \mathrm{mg}$ of Ticagrelor twice daily were prescribed. Other standard drugs (angiotensin converting enzyme inhibitors, beta blockers, statins and oral or injectable hypoglycemic agents) remained unchanged during the study in order to minimize the effects of alterations on the echocardiographic variables.

\section{Statistical analysis}

Data were processed and analysed using SPSS (Statistical Package for Social Sciences), version 25.0. Test statistics used to analyze the data were descriptive statistics, chisquare $\left(\chi^{2}\right)$ and unpaired t-test. Data presented on categorical scale were compared between groups using chi-square $\left(\chi^{2}\right)$, while data presented on continuous scale and normally distributed were compared between groups with the help of unpaired t-test. Percentage changes in all of the echocardiographic variables from baseline to 3 months following PCI were determined. These variables were then compared between the two groups using unpaired t-test. For analytical tests level of significance was set at $5 \%$ and p-value $<0.05$ was considered significant. Sample size was calculated from the values of previous study. ${ }^{14}$

\section{Result:}

The number of patients enrolled in each group was 37 . During follow up 7 patients from diabetic and 3 from non-diabetic groups lost leaving 30 in diabetic and 34 in non-diabetic groups for final analysis. More than half $(56.7 \%)$ of the subjects in the diabetic group and twothirds $(67.6 \%)$ in the non-diabetic group were 50 or $<$ 50 years old with no significant intergroup difference $(p$ $=0.365)$. In terms of gender distribution, a male predominance was observed in both group $(p=0.386)$ (table I).
Table-I

Distribution of patients by their demographic characteristics

\begin{tabular}{lccc}
\hline Demographics $^{*}$ & \multicolumn{2}{c}{ Group } & p-value \\
\cline { 2 - 3 } & $\begin{array}{c}\text { Diabetic } \\
(\mathrm{n}=30)\end{array}$ & $\begin{array}{c}\text { Non-diabetic } \\
(\mathrm{n}=34)\end{array}$ & \\
\hline Age (years) & & & \\
$\leq 50$ & $17(56.7)$ & $23(67.6)$ & 0.365 \\
$>50$ & $13(43.3)$ & $11(32.4)$ & \\
Sex & & & \\
Male & $21(70.0)$ & $27(79.4)$ & \\
Female & $9(30.0)$ & $7(20.6)$ & 0.386 \\
\hline
\end{tabular}

Figures in the parentheses indicate corresponding \%; *Chi-squared test $\left(C^{2}\right)$ was done to analyze the data.

Diabetic group had a significantly higher mean fasting blood sugar than the non-diabetic group $(p<0.001)$. The mean $\mathrm{HbAlc}$ was also significantly higher in the former group than that in the latter group $(\mathrm{p}<0.001)$. However, none of the serum lipids shown in table were any different between the study groups $(\mathrm{p}>0.05)$ (table II).

Table-II

Distribution of patients by their laboratory investigation findings

\begin{tabular}{lccc}
\hline $\begin{array}{l}\text { Laboratory } \\
\text { investigations }\end{array}$ & \multicolumn{2}{c}{ Group } & p-value \\
\cline { 2 - 3 } & $\begin{array}{c}\text { Diabetic } \\
(\mathrm{n}=30)\end{array}$ & $\begin{array}{c}\text { Non-diabetic } \\
(\mathrm{n}=34)\end{array}$ & \\
\hline FBS (mmol/L) & $9.3 \pm 2.0$ & $5.5 \pm .07$ & $<0.001$ \\
HbA1c (\%) & $7.5 \pm 1.0$ & $4.9 \pm 0.5$ & $<0.001$ \\
S. Cholesterol (mg/dl) & $188.0 \pm 42.5$ & $200.5 \pm 50.8$ & 0.292 \\
LDL (mg/dl) & $127.1 \pm 27.6$ & $136.4 \pm 32.2$ & 0.219 \\
HDL (mg/dl) & $36.5 \pm 5.2$ & $35.5 \pm 4.7$ & 0.424 \\
Triglycerides (mg/dl) & $230.7 \pm 59.1$ & $212.9 \pm 69.3$ & 0.276 \\
\hline
\end{tabular}

\#Data were analyzed using unpaired t-test and were presented as mean $\pm \mathrm{SD}$.

Before PCI, left ventricular ejection fraction (LVEF) was on an average $1.6 \%$ lower in diabetic group than that in non-diabetic group $(p=0.070)$. Number of segments with abnormal wall motion (WM) was much higher in the diabetics than that in the non-diabetics $(p=0.014)$. While the left ventricular end diastolic volume (LVEDV), the left ventricular end systolic volume (LVESV) was no different between the groups $(p=0.076$ and $p=0.368$ respectively). Left ventricular internal dimension in diastole (LVIDd) and left ventricular internal dimension in systole (LVIDs) were also higher in the diabetics than those in the non-diabetics $(\mathrm{p}<0.001$ and $\mathrm{p}=0.046$ respectively) (table III). 
After 3 months of PCI, LVEF increased in both groups \& from their baseline figures with number of abnormal WM segments decreased. LVEDV decreased further with decrease being more pronounced in the non-diabetic group so the two groups became almost identical in terms this variable $(p=0.221)$. LVESV and LVIDd both further decreased in either group maintaining significant difference between the groups with respect to these variables as before $(\mathrm{p}=0.017$ and $\mathrm{p}=0.008$ respectively). However, LVIDs did not respond much and the difference between the groups in terms of this variable remained insignificant $(p=0.060)$ (table IV).

\section{Table-III}

Echocardiographic evaluation of left ventricular systolic function before PIC

\begin{tabular}{lccc}
\hline \multirow{2}{*}{$\begin{array}{l}\text { Left ventricular } \\
\text { systolic function } \\
\text { parameters }\end{array}$} & \multicolumn{2}{c}{ Group } & p-value \\
\cline { 2 - 3 } & $\begin{array}{c}\text { Diabetic } \\
(\mathrm{n}=30)\end{array}$ & $\begin{array}{c}\text { Non-diabetic } \\
(\mathrm{n}=34)\end{array}$ & \\
\hline $\begin{array}{l}\text { LVEF (\%) } \\
\text { No. of segments with }\end{array}$ & $53.9 \pm 3.8$ & $55.5 \pm 4.7$ & 0.070 \\
abnormal WM & $2 \pm 1$ & $1 \pm 1$ & 0.014 \\
LVEDV (ml) & $96.0 \pm 8.1$ & $94.4 \pm 8.4$ & 0.076 \\
LVESV (ml) & $44.3 \pm 2.9$ & $42.1 \pm 3.5$ & 0.368 \\
LVIDd (mm) & $51.9 \pm 4.5$ & $49.4 \pm 3.4$ & $<0.001$ \\
LVIDs (mm) & $35.1 \pm 5.4$ & $32.6 \pm 4.5$ & 0.046 \\
\hline
\end{tabular}

\#Data were analyzed using unpaired t-test and were presented as mean $\pm \mathrm{SD}$.

\section{Table-IV}

Echocardiographic evaluation of LV systolic function 3 months after PIC

\begin{tabular}{lccc}
\hline $\begin{array}{l}\text { LV systolic function } \\
\text { parameters }^{\#}\end{array}$ & \multicolumn{2}{c}{ Group } & p-value \\
\cline { 2 - 3 } & $\begin{array}{c}\text { Diabetic } \\
(\mathrm{n}=30)\end{array}$ & $\begin{array}{c}\text { Non-diabetic } \\
(\mathrm{n}=34)\end{array}$ & \\
\hline LVEF (\%) & $57.8 \pm 2.3$ & $59.3 \pm 2.6$ & 0.027 \\
No. of segments & $1 \pm 1$ & $0.03 \pm 0.02$ & $<0.001$ \\
with WMA & & & \\
LVEDV (ml) & $74.9 \pm 22.7$ & $68.3 \pm 19.5$ & 0.221 \\
LVESV (ml) & $31.6 \pm 10.5$ & $27.8 \pm 8.7$ & 0.017 \\
LVIDd (mm) & $50.2 \pm 2.3$ & $48.1 \pm 2.6$ & 0.008 \\
LVIDs (mm) & $33.1 \pm 2.6$ & $31.2 \pm 4.8$ & 0.060 \\
\hline
\end{tabular}

\#Data were analyzed using unpaired t-test and were presented as mean $\pm \mathrm{SD}$.

Changes in LV function parameters from baseline to 3 months after PCI shows that there is no difference between diabetics and non-diabetics in terms of percentage of improvement in LVEF, percentage of decrease in LVEDV, LVESV and LVIDs $(p=0.631, p=$ 0.657 and $p=0.088$ respectively). The percentage of decrease in WMA LVIDd, LVIDs are no difference between diabetic and non diabetic $\operatorname{group}(\mathrm{p}=0.061, \mathrm{p}=$ 0.210 and $p=0.201$ respectively) (table $\mathrm{V}$ ).
Table-V

Difference in LV function parameters from baseline to 3 months after PCI in DM Vs non DM

\begin{tabular}{lccc}
\hline $\begin{array}{l}\text { LV systolic } \\
\text { function parameters }\end{array}$ & \multicolumn{2}{c}{ Group } & p-value \\
\cline { 2 - 3 } & $\begin{array}{c}\text { Diabetic } \\
(\mathrm{n}=30)\end{array}$ & $\begin{array}{c}\text { Non-diabetic } \\
(\mathrm{n}=34)\end{array}$ & \\
\hline $\begin{array}{l}\text { \% of increase in } \\
\text { LVEF (\%) }\end{array}$ & $8.4 \pm 1.2$ & $7.9 \pm 1.2$ & 0.631 \\
$\begin{array}{l}\text { \% of decrease in no. of } \\
\text { segments with WMA }\end{array}$ & $86.7 \pm 8.0$ & $96.0 \pm 4.0$ & 0.061 \\
$\begin{array}{l}\text { \% of decrease in } \\
\text { LVEDV (ml) }\end{array}$ & $22.6 \pm 3.6$ & $24.8 \pm 3.3$ & 0.657 \\
\% of decrease in & $26.6 \pm 4.1$ & $29.1 \pm 3.1$ & 0.088 \\
$\begin{array}{l}\text { LVESV (ml) } \\
\text { \% of decrease in }\end{array}$ & $3.6 \pm 1.0$ & $3.9 \pm 1.4$ & 0.210 \\
$\begin{array}{l}\text { LVIDd (mm) } \\
\text { \% decrease in }\end{array}$ & $5.7 \pm 1.9$ & $4.8 \pm 1.1$ & 0.201 \\
LVIDs (mm) & & & \\
\hline
\end{tabular}

\#Data were analyzed using unpaired t-test and were presented as mean \pm SD.

\section{Discussion:}

The study was intended to compare the changes in left ventricular systolic function from baseline to 3 months after PCI between diabetic and non-diabetic patients.

In this study we found, diabetic patients have significant LAD stenosis $(60 \%)$ than non diabetics $(41.2 \%)(\mathrm{p}=$ 0.133 ). The significant stenosis in the RCA and LCx were however, almost identical between groups ( $p=0.683$ and $\mathrm{p}=0.909$ respectively.

All patients were assessed by $2 \mathrm{D}$ echocardiography before PCI, at discharge \& 3 months after PCI. At baseline, diabetic patients had LVEF $53.9 \pm 3.8 \%$ where as non diabetics had $55.5 \pm 4.7 \%$, but the difference was not statistically significant $(p=0.070)$ though baseline parameter in diabetics was low. There was also no significant difference in LVESV and LVIDs in between DM \& non DM group $(\mathrm{p}=0.368, \mathrm{p}=0.06$ respectively). Regional wall motion abnormality was present more in diabetics than non diabetics $(\mathrm{p}=0.014)$.

On follow up 3 months after PCI again we assessed by 2D echocardiography and found that, there was improvement of all parameters of LV systolic function in both diabetic \& non diabetic group. From baseline to 3 months after PCI LVIDs decreased in both diabetics $5.7 \pm 1.9 \%$ and non diabetics $4.8 \pm 1.1 \%$ but the difference between these two goups is not significant $(p=0.201)$.

Nabati et al. $2016^{15}$ found that, in diabetic group baseline \& 1 month after PCI LVIDs was $31 \pm 6.3 \& 28 \pm 5.8$ 
respectively and $10 \%$ decrement occurred of LVIDs ( $p$ $=0.002$ ) in this group. Where as in non diabetic group baseline \& 1 month after PCI LVIDs was $28 \pm 4.1$ \& $30.9 \pm 6.6$ respectively and $8 \%$ increment occur of LVIDs. Our study is consistent with this findings. Though, their study have 1 month follow up period and our study have 3 months. So, we can say our study is more reliable.

In our study, we found, more regional wall motion abnormality(WMA) in diabetic $2 \pm 1$ than non diabetic groups $1 \pm 1(\mathrm{p}=0.014)$ at baseline. 3 months after PCI WMA in diabetics $1 \pm 1$ and in non diabetic $0.03 \pm 0.02(\mathrm{p}$ $=<0.001)$ and WMA decreased $86.7 \pm 8.0 \%$ in diabetic where as $96.0 \pm 4 \%$ decreased in non diabetics $(\mathrm{P}=0.061)$.

In nabati et al. $2016^{15}$, before PCI, the WMA was observed in $2.2 \pm 2.91$ segments that significantly decreased to $1.5 \pm 2.58$ segments $(p=0.04)$ after the procedure. However, there was no significant difference in WMA improvement between the diabetics and nondiabetics, (before PCI: 2.69 \pm 2.96 and 1.77 \pm 2.86 and after PCI: $1.69 \pm 2.9$ and $1.33 \pm 2.24$ segments respectively $(\mathrm{p}=0.4)$.

In our study, from baseline to 3 months after PCI LVEF improved $8.4 \pm 1.2 \%$ in diabetics and $7.9 \pm 1.2 \%$ in non diabetics but the difference of this improvement between two groups is not statistically significant $(p=0.631)$. Nabati et al. $2016^{15}$, found that 1 month after PCI diabetic patients improved in LVEF, but non diabetic patient didn't show any significant improvement in LVEF $(p=0.004)$

In 2008, Mehrpooyaet al. ${ }^{9}$ assessed the effects of PCI on LVEF and WMA in 21 patients who presented with ischemic cardiac chest pain, an ejection fraction less than $40 \%$, and significant coronary occlusion (70\%). Echocardiography was performed at baseline and 1 month after the procedure. The mean EF increased significantly after angioplasty $(p<0.000)$. All of the patients $(100 \%)$ had wall motion abnormality at baseline, but the frequency decreased to $65 \%$ of them after the procedure. The improvement of EF in patients with significant stenosis of the LAD was higher than in patients without LAD lesions $(\mathrm{p}=0.008)$.

In 2005, Agirbasli et al. ${ }^{16}$ evaluated LV contractility and myocardial perfusion after PCI in 60 patients who underwent successful LAD stenting. Myocardial perfusion and LVEF improved at $6 \pm 3$ months after the procedure $(\mathrm{p}=0.05)$. Patient-related factors, such as DM, presentation with acute coronary syndrome and age, did not affect the LVEF change after the procedure.
In our study, diabetics showed significant improvement in the systolic function such as LVIDd, LVIDs, LVEDV, LVSDV and LVEF as almost equal to non-diabetics. At baseline, most of these parameters were worse in diabetics compared to non-diabetics. This outcome may be due to a worse effect of diabetes on ischemia. However, our study also showed excellent reversibility of these adverse effects after PCI in this subgroup. In non-diabetics, there was also change in LVEF after PCI. WMA improvement was observed in both diabetics and non-diabetics. Though, there was varing degree of improvement in this two groups. For such variation, the worse baseline echocardiographic parameters in diabetic patients may be the cause.

Basic three mechanism of restenosis are (a) Intimal elastic recoil (b) late remodeling (c) Intimal hyperplasia. Stents control first two of the three mechanisms of restenosis ${ }^{5}$ and the introduction of stents that release drugs with inhibition effects on intimal hyperplasia may reduce or eliminate the main limitation of angioplasty. ${ }^{17}$ Polymer-based drug-eluting stents have been shown to significantly reduce angiographic restenosis in comparison with bare metal stents ${ }^{18}$ Lower probability of repeat revascularization and stent thrombosis with DES have influenced selected options of revascularization for diabetic patients. This is particularly useful with the newer generation of DES. ${ }^{19}$

In 2005 Roffi $^{7}$ described that, diabetic patients with nonST-segment elevation acute coronary syndromes are at high risk for subsequent cardiovascular events. At the same time, however, they derive greater benefit than nondiabetic counter parts from an aggressive acute phase management based on platelet glycoprotein IIb/IIIa receptor antagonists and an early invasive strategy. Despite the benefit, these treatment modalities remain underutilized among patients with diabetes mellitus. The widespread use of DES will further improve outcomes in patients with diabetes undergoing early percutaneous revascularization.

In 2005, Windeker et al. ${ }^{20}$ showed the impact of drugeluting stents on restenosis has been dramatic. The broad use of these devices is expected to markedly improve the outcomes of diabetic patients with ACS undergoing early invasive strategy. Recent data suggest that the sirolimus-eluting stent (Cypher, Cordis, Miami, FL, USA) is superior to the paclitaxeleluting stent (Taxusw, Boston Scientific, Natick, MA, USA) among individuals with diabetes. Accordingly, a pre-specified subgroup analysis of the diabetic population (n 1/4 201) of the Swiss 
randomized SIRTAX (Sirolimus Versus Taxus) trial showed a significant decrease in the incidence of death, MI, or ischemia-driven target lesion revascularization at 9 months in the sirolimus stent group (hazard ratio, 0.31, $95 \%$ confidence interval, 0.12 to 0.78 ). Similarly recent data suggest that newer generation of DES gives better outcome than earlier generation of DES.

Our study demonstrated that improvement of the parameters of left ventricular systolic function after using of drug eluting stent in diabetic patients with NSTEMI was not inferior to the non diabetic group under same condition. So, indications of PCI with drug eluting stent may be extended in diabetic patient with NSTEMI.

\section{This study has the following limitations:}

- Seven patients from the diabetic group and three patients from the non-diabetic group were lost to follow up, which might have affected the outcome of our study.

- As the study was a part of academic course, the duration of follow up was limited due to time bar.

- Not all parameters of the left ventricular systolic function assessment were measured, only most validated recommended ones from 2017 ESC recommendation for standarization of adult thorasic echocardiography reporting in agreement with recent chamber quantification are used.

So, further study with long duration of follow up is needed.

\section{Conflict of interest:}

Author has no conflict of interest

\section{Acknowledgments:}

This study was a postgraduate thesis. The authors would like to thank all of the patients who enrolled in this study and staff of the Department of Cardiology, University Cardiac Center, Bangabandhu Sheikh Mujib Medical University, Dhaka, Bangladesh. Financial support was obtained from the Research Council of the Bangabandhu Sheikh Mujib Medical University, Dhaka, Bangladesh.

\section{References:}

1. White HD, Chew DP, Acute myocardial infarction. Lancet 2008; 372(9638):570 84 .

2. Terkelsen CJ, Lassen JF, Nørgaard BL, Gerdes JC, Jensen T, Gøtzsche LB, et al.Mortality rates in patients with ST elevation vs. non ST elevation acute myocardial infarction: Observations from an unselected cohort. Eur Heart J 2005;26:18 26.

3. Boden WE.Management of patients with diabetes and coronary artery disease. In: Sobel BE, Schneider DJ, editors. Medical Management of Diabetes and Heart Disease. New York: Marcel Dekker; 2002. p.185-209
4. Banning AP, Westaby S, Morice MC, Kappetein AP, Mohr FW, Berti S. et al. Diabetic and nondiabetic patients with left main and/ or 3-vessel coronary artery disease: comparison of outcomes with cardiac surgery and paclitaxel-eluting stents. J Am Coll Cardiol 2010;55(11):1067-75.

5. Creager MA, Luscher TF, Cosentino F, Beckman JA. Diabetes and vascular disease: pathophysiology, clinical consequences, and medical therapy: Part I. Circulation 2003;108(12): 1527-32.

6. Roffi M, Dominick J. Angiolillo, and A. Pieter Kappetein. Current concepts on coronary revascularization in diabetic patients. Euro Heart J 2011;32:2748-57.

7. Roffi M,Early invasive strategy in thediabetic patient with non-STsegment elevation acute coronary syndromes. Euro Heart J2005;7Supplement K:K19-K22

8. Alonso JJ, Dura'n JM, Gimeno F, Ramos B, Serrador A, Ferna'ndezAvile F,Coronary angioplasty in diabetic patients. Current and future perspectives. Rev Esp Cardiol 2002;55(11):1185-200.

9. Mehrpooya M, Ghasemi M, Masud, Shahrzad I, Improvement in left ventricular ejection fraction and wall motion abnormality after successful angioplasty and stenting of chronic coronary obstruction. Heart, Lung and Ciculation 2008; 17supplement 1:S32.

10. Chobanian AV, Bakris GL, Black HR, et al. Seventh report of the Joint National Committee on Prevention, Detection, Evaluation, and Treatment of High Blood Pressure. Hypertension 2003;42: 1206-52.

11. American Diabetes Association.Classification and Diagnosis aof Diabetes Mellitus: Standards of Medical Care in Diabetes-2018. Diabetes Care 2018;41supplement 1:S13-S27.

12. Parmar MS, Family history of coronary artery disease - need to focus on proper definition!. Eur Heart J 2003;24(22):2073.

13. Sasao H, Endo A, Hasegawa T, Oimatsu H, Takada T, Comparison of three-year clinical outcome of the multi-link stent with the PalmazSchatz stent in Japanese patients with coronary artery disease: a case control study. J Invasive Cardiol2003;15(8):433-36

14. Acar B, Ozeke O, Unal S, Karakurt M, Kera M, Kirbas O, et al. Change in the left ventricular systolic function in patients with ST elevation myocardial infarction: Evedence for smoker's paradox or pseudo paradox?. Ind. Heart J 2016;68(6):816-20.

15. Nabati M, Taghavi M, Saffar N, Yazdani J, Bagheri B, Alteration of echocardiographic left ventricular function after percutaneous coronary stenting in diabetic patients with isolated severe proximal left anterior descending artery stenosis. Indian Heart J2017;69(2):146-50.

16. Agirbasli M, Guler N, Recovery of left ventricular systolic function after left anterior descending coronary artery stenting. J Interv Cardiol2005;18(2):83-8.

17. Regar E, Serruys PW, The Ravel trial. Zero percent restenosis: a cardiologists dream comes true!. Rev Esp Cardiol 2002;55(5): 459-62.

18. Stone GW, Midei M, Newman W, Sanz M, Hermiller JB, Williams J, et al. Randomized comparison of everolimus- eluting and paclitaxeleluting stents: two-year clinical follow-up from the Clinical Evaluation of the Xience V Everolimus Eluting Coronary Stent System in the Treatment of Patients with de novo Native Coronary Artery Lesions (SPIRIT) III trial. Circulation2009;119(5):680-86.

19. Silvain J, Vignalou JB, Barthe' le'my O, Kerneis M, Collet JP, Montalescot G, Coronary revascularization in the diabetic patient', Circulation 2014;130:918-22.

20. Windecker S, Remondino A, Eberli FR et al. 'Sirolimus-eluting and paclitaxel-eluting stents for coronary revascularization. N Engl J Med 2005;353:653-62 